The University of Southern Mississippi

The Aquila Digital Community

Faculty Publications

6-1-1997

\title{
Numerical Modeling of Self-Propagating Polymerization Fronts: The Role of Kinetics on Front Stability
}

\author{
Stanislav E. Solovyov \\ University of Southern Mississippi \\ Victor M. Ilyashenko \\ University of Southern Mississippi \\ John A. Pojman \\ University of Southern Mississippi
}

Follow this and additional works at: https://aquila.usm.edu/fac_pubs

Part of the Chemistry Commons

\section{Recommended Citation}

Solovyov, S. E., llyashenko, V. M., Pojman, J. A. (1997). Numerical Modeling of Self-Propagating Polymerization Fronts: The Role of Kinetics on Front Stability. Chaos, 7(2), 331-340.

Available at: https://aquila.usm.edu/fac_pubs/5327

This Article is brought to you for free and open access by The Aquila Digital Community. It has been accepted for inclusion in Faculty Publications by an authorized administrator of The Aquila Digital Community. For more information, please contact Joshua.Cromwell@usm.edu. 


\title{
Numerical modeling of self-propagating polymerization fronts: The role of kinetics on front stability
}

\author{
Stanislav E. Solovyov, Victor M. Ilyashenko, and John A. Pojman \\ Department of Chemistry and Biochemistry, University of Southern Mississippi, Hattiesburg, \\ Mississippi 39406-5043
}

(Received 18 November 1996; accepted for publication 14 February 1997)

\begin{abstract}
Frontal propagation of a highly exothermic polymerization reaction in a liquid is studied with the goal of developing a mathematical model of the process. As a model case we consider monomers such as methacrylic acid and $n$-butyl acrylate with peroxide initiators, although the model is not limited to these reactants and can be applied to any system with the similar basic polymerization mechanism. A three-step reaction mechanism, including initiation, propagation and termination steps, as well as a more simple one-step mechanism, were considered. For the one-step mechanism the loss of stability of propagating front was observed as a sequence of period doubling bifurcations of the front velocity. It was shown that the one-step model cannot account for less than $100 \%$ conversion and product inhomogeneities as a result of front instability, therefore the three-step mechanism was exploited. The phenomenon of superadiabatic combustion temperature was observed beyond the Hopf bifurcation point for both kinetic schemes and supported by the experimental measurements. One- and two-dimensional numerical simulations were performed to observe various planar and nonplanar periodic modes, and the results for different kinetic schemes were compared. It was found that stability of the frontal mode for a one-step reaction mechanism does not differ for 1-D and 2-D cases. For the three-step reaction mechanism 2-D solutions turned out to be more stable with respect to the appearance of nonplanar periodic modes than corresponding 1-D solutions. Higher Zeldovich numbers (i.e., higher effective activation energies or lower initial temperatures) are necessary for the existence of planar and nonplanar periodic modes in the 2-D reactor with walls than in the 1-D case. ᄀ (C) 1997 American Institute of Physics. [S1054-1500(97)00202-4]
\end{abstract}

\begin{abstract}
Autocatalytic reactions have long been known to support a constant velocity wave front resulting from the coupling of diffusion and chemical reaction, with a common example being the reaction front of an ordinary flame. In general, this type of self-propagating reaction front consists of (1) the ignition step, where some form of energy (e.g., heat, light) is applied to initiate the reaction, followed by (2) the diffusion of heat and reactive radicals into unreacted regions, inducing the reaction there. For a reaction front to exist, the chemical reaction must be exothermic, with a fairly high activation energy. In this work, frontal propagation of a highly exothermic polymerization reaction in a liquid was studied experimentally with the goal of developing a realistic model. The models examined included either one or three chemical reaction steps, and it was found that the stability of the wave front for the one-step model was the same for both one- and two-dimensional geometries. However, for the more realistic three-step reaction model, the two-dimensional case was more stable than the one-dimensional case.
\end{abstract}

\section{INTRODUCTION}

Propagating fronts of autocatalytic reactions have been observed in gaseous, liquid, and solid phases. An example of a successful application of propagating fronts in solids is the self-propagating high-temperature synthesis (SHS) of advanced materials. ${ }^{1,2}$ Frontal propagation of addition polymerization is an example of a chemical reaction in condensed phase. ${ }^{3-5}$ Depending upon the experimental conditions (e.g., adiabatic propagation, the presence of heat losses, or applied pressure) the reaction proceeds in liquid phase only, or may involve the solidification of the resulting polymer. In both cases propagating fronts resemble gaseous flames and SHS because they both involve the thermal propagation mechanism. Free radical polymerization reactions are usually highly exothermic, and the heat of the reaction provides autocatalysis for a polymerization front propagating through a liquid monomer. Model systems most commonly used in frontal polymerization processes include such monomers as methacrylic acid (MA), $n$-butyl acrylate (BA), methyl methacrylate $\neg(\mathrm{MMA}), \neg$ triethylene $\neg$ glycol $\neg$ dimethacrylate (TGDMA), etc., and initiators such as benzoyl peroxide (BPO), tert-butyl peroxide (tBPO), lauroyl peroxide (LPO) and 2,2' -azo-bis-isobutyronitrile (AIBN). Commercial applications of these processes (such as synthesis of phaseseparated materials ${ }^{6}$ ) may come from the high-energy efficiency and low environmental impact of the technique. ${ }^{7}$

Polymer fronts have been studied at the Institute of Chemical Physics (Chernogolovka), Russia since the early 1970s in tubular chemical reactors under high pressure., More recently frontal regimes in an unmoving medium for various monomer+initiator systems were studied under am- 
bient conditions ${ }^{5,8,9}$ High-viscosity modifiers were also used in our lab experiments to suppress convective instabilities, and high pressures were used to prevent bubbles that affect the front velocity and may lead to front decay. ${ }^{10}$ In recent years a few modifications of the technique such as cylindrical and spherical fronts in plug-flow reactors were proposed to avoid difficulties of maintaining stable front propagation arising from formation of jet flows and inherit thermal instabilities. ${ }^{11-13}$ Under certain conditions thermal instabilities in plug-flow reactors combined with hydrodynamic effects are known to result in a transition of frontal propagation $\neg$ mode $\neg$ to $\neg$ low-temperature $\neg$ homogeneous polymerization. ${ }^{11,12}$

The theory of similarity of temperature and concentration fields allowed Zeldovich and Frank-Kamenetsky to develop an approximate theory of slow combustion for such systems $^{14-16}$ in 1938 . The normal velocity of front propagation in an unmoving condensed medium was calculated by Novozhilov ${ }^{17}$ using the infinitely narrow reaction zone approximation, and an explicit formula for it was proposed for first- and second-order reactions. By using this formula the effective activation energy of a polymerization reaction can be calculated by measuring the velocity of front propagation at different initial temperatures and assuming the thermal diffusivity is temperature independent.

Recently, our experiments demonstrated much more complex behavior of the system than that anticipated in early works. ${ }^{8,9,18}$ This includes convective effects inherit to liquid polymerizing systems and the effects of the materials' physical properties such as monomer boiling point, viscosity of monomer and polymer phases on the front existence, and hydrostatic stability. All these factors could not be accounted for in simple earlier models. Thus, a mathematical model adequately describing such a system must include realistic polymerization kinetics, thermal diffusion, and appropriate hydrodynamics if convective liquid motion is involved. In this paper we take a step in that direction and develop the mathematical model with three-step polymerization kinetics and heat diffusion, not only reproducing experimental results but also predicting the qualitative system behavior and some properties of the final product in the absence of convection.

\section{EXPERIMENTAL}

The experimental setup for studying polymerization fronts consists of a vertical glass tube filled with a solution of methacrylic acid (MA) and a thermal initiator such as benzoyl peroxide (BPO) at room temperature (other polymerizing systems were also used). The front is triggered by applying a heat source to the top of the tube for a short period of time. The autocatalytic polymerization reaction propagates through the solution of the monomer and initiator as a wave front leaving the solid polymer behind. To control the initial temperature and homogeneous reaction in the cold zone, the tube was placed into a water bath at a fixed temperature and slowly withdrawn to maintain the constant clearance between the water surface and the reaction front as the polymerization front proceeded. ${ }^{10}$ The front velocity and tem- perature $\neg$ profiles $\neg$ were $\urcorner$ measured $\neg$ during $\urcorner$ the $\neg$ front propagation, and the polymer obtained was analyzed. ${ }^{9,19}$ At some experimental conditions front instabilities such as velocity pulsations and spin modes were observed. ${ }^{9}$

\section{KINETIC MODEL}

For the model purposes an adiabatic unstirred reactor is considered. The rate of the radical generation is considered to be negligible at the initial temperature. Diffusion of the radicals is neglected because thermal diffusion in such polymerization systems is faster than mass diffusion by at least 100 times.

The simplified kinetic model of the addition polymerization with thermal initiation has the following form:

$$
\begin{aligned}
& \left.I+\Delta \rightarrow 2 R,\urcorner \quad k_{d}=A_{d} \exp \left(-E_{a, d} / R_{g} T\right),\right\urcorner \\
& \left.\left.R+M \rightarrow P_{1}+\Delta,\right\urcorner \quad k_{p}=A_{p} \exp \left(-E_{a, p} / R_{g} T\right),\right\urcorner \\
& \left.\left.P_{n}+M \rightarrow P_{n+1}+\Delta,\right\urcorner \quad k_{p},\right\urcorner \\
& \left.\left.P_{n}+R \rightarrow P_{\text {dead }}+\Delta,\right\urcorner \quad k_{t}=A_{t} \exp \left(-E_{a, t} / R_{g} T\right),\right\urcorner \\
& \left.\left.P_{n}+P_{m} \rightarrow P_{\text {dead }}+\Delta,\right\urcorner k_{t} .\right\urcorner
\end{aligned}
$$

Here $I$ is the initiator, $R$ denotes the primary radicals, $M$ is the monomer, $P_{n}$ is the growing polymer chain, $P_{\text {dead }}$ is the dead polymer, $k_{d}, k_{p}, k_{t}$ are corresponding temperature dependent rate constants for the reactions shown, $E_{a, d}, E_{a, p}$, $E_{a, t}$ are the activation energies for these reactions and $A_{d}$, $A_{p}, A_{t}$ are corresponding preexponents; $\Delta$ is the reaction heat release, $R_{g}$ is the universal gas constant, and $T$ is the temperature,

\section{MATHEMATICAL MODEL}

We consider the heat release during the first propagation step (2) and termination steps (4) and (5) to be negligible compared to the propagation step (3). That is certainly true for our systems. Then the nonstationary two-dimensional equations describing the kinetics of the process and the heat balance for the system (1)-(5) have the following form:

$$
\begin{aligned}
& \left.\frac{d[I]}{d t}=-k_{d}[I],\right\urcorner \\
& \left.\frac{d[R]}{d t}=2 k_{d} f[I]-k_{p}[R][M]-k_{t}[R][P \cdot]-k_{t}[R]^{2},\right\urcorner \\
& \frac{d[M]}{d t}=-k_{p}[R][M]-k_{p}[M][P \cdot], \neg \\
& \left.\frac{d[P \cdot]}{d t}=k_{p}[R][M]-k_{t}[R][P \cdot]-k_{t}[P \cdot]^{2},\right\urcorner \\
& \left.\frac{d[P]}{d t}=k_{t}[R][P \cdot]+k_{t}[P \cdot]^{2},\right\urcorner \\
& \frac{\partial T}{\partial t}=\kappa\left(\frac{\partial^{2} T}{\partial x^{2}}+\frac{\partial^{2} T}{\partial y^{2}}\right)-\frac{\Delta H}{\rho c_{p}} k_{p}([M][R]+[M][P \cdot]) .
\end{aligned}
$$


Here, the reaction enthalpy $\Delta H=-13.8 \mathrm{kcal} \mathrm{mol}^{-1}$, the monomer density $\rho=1038 \mathrm{~g} \mathrm{~L}^{-1}$, the monomer heat capacity $\left.\neg c_{p}=0.4 \mathrm{cal} \mathrm{g}^{-1} \mathrm{~K}^{-1},\right\urcorner$ the $\urcorner$ thermal $\neg$ diffusivity $\kappa$ $=0.0014 \mathrm{~cm}^{2} \mathrm{~s}^{-1}$, the initiator efficiency $f=0.5$, and all concentrations are in mol L' $\mathrm{L}^{-1}:[I]$ is the initiator concentration, $[R]$ is the concentration of primary radicals, $[M]$ is the monomer concentration, $[P \cdot]$ is the concentration of polymer radicals, and $[P]$ is the dead polymer concentration.

\section{INITIAL AND BOUNDARY CONDITIONS}

For $0 \leqslant x \leqslant L,-r \leqslant y \leqslant r$ where $x$ is the coordinate along the tube axis in the direction of front propagation and $y$ is the radial coordinate in the cross section of a cylinder, the initial conditions are

$$
\begin{aligned}
& {[R]_{0}=0,} \\
& {[M]_{0}=6 \mathrm{~mol} \mathrm{~L}^{-1},} \\
& {[P \cdot]_{0}=0,} \\
& {[P]_{0}=0,}
\end{aligned}
$$

for $x=0: T_{i}=T_{0}+\Delta T,[I]_{0}=0$ (the ignition zone, $\Delta T$ $=150-200 \mathrm{~K}) ; \neg$ for $\neg 0<x \leqslant L: \neg T_{0}=250-320 \mathrm{~K}, \neg[I]_{0}$ $=0.002-0.1 \mathrm{~mol} \mathrm{~L}^{-1}$. The initial temperature $T_{0}$ and the initiator concentration $[I]_{0}$ were varied depending on the conditions and the initiator used.

Adiabatic boundary conditions at both tube ends and walls are assumed:

$$
\begin{aligned}
& d T / d x=0\urcorner \text { for } x=0, x=L,\urcorner \\
& d T / d y=0\urcorner \text { for } y=-r, y=r .\urcorner
\end{aligned}
$$

The tube radius $r=1-2 \mathrm{~cm}$, and the tube length $L$ $=15-30 \mathrm{~cm}$.

\section{COMBINED CONCENTRATION OF FREE RADICALS}

In deriving the system (6)-(11) we assumed that the rate constants $k_{p}$ for reactions of primary radicals (from the initiator) and growing polymer chains with the monomer are the same. It is an acceptable assumption for long polymer radicals where the propagation rate constant does not change significantly with the length of the polymer if the gel effect can be neglected. However, it is obviously not true when the primary and polymer radicals are compared because of their different chemical nature. While there are no widely available data on the reaction rate constants of primary radicals with the monomer for our systems, we can eliminate this problem considering the combined concentration of the free radicals:

$$
[\widetilde{R}]=[R]+[P \cdot] \cdot \neg
$$

It is possible to do so because the reaction frequency of the primary radicals with the monomer is much lower than that of the polymer radicals. For example, the average degree of polymerization of MA with BPO in the frontal regime at ambient conditions ${ }^{19}$ was estimated to be 25 . It means that even for the low molecular weights obtained, 24 addition reactions take place per one reaction of the BPO primary radical with the monomer. Then, the effect of this reaction on the overall propagation step kinetics does not exceed $4 \%$ even if its rate differs significantly $\left(0.5 k_{p}<k_{\text {primary }}<\infty\right.$ and even broader range for higher molecular weights) and can therefore be neglected. Recent experimental studies of propagation $\neg$ step $\neg$ kinetics $\neg$ of $\neg$ methyl $\neg$ methacrylate $\neg$ and methacrylonitrile ${ }^{20}$ demonstrated that the rate constant of the first propagation step $k_{\text {primary }} \equiv k_{p 1}$ is $4-6$ times greater than $k_{p} \equiv k_{p \infty}$ at $60^{\circ} \mathrm{C}$. Considering this, we used the combined concentration of free radicals in our simulations for the three-step reaction mechanism. Then the nonstationary twodimensional equations corresponding to Eqs. (6)-(11) take the following form:

$$
\begin{aligned}
& \frac{d[I]}{d t}=-k_{d}[I], \neg \\
& \left.\frac{d[\widetilde{R}]}{d t}=2 k_{d} f[I]-k_{t}[\widetilde{R}]^{2},\right\urcorner \\
& \frac{d[M]}{d t}=-k_{p}[\widetilde{R}][M], \neg \\
& \frac{d[P]}{d t}=k_{t}[\widetilde{R}]^{2}, \neg \\
& \frac{\partial T}{\partial t}=\kappa\left(\frac{\partial^{2} T}{\partial x^{2}}+\frac{\partial^{2} T}{\partial y^{2}}\right)-\frac{\Delta H}{\rho c_{p}} k_{p}[M][\widetilde{R}] . \neg
\end{aligned}
$$

In the following sections the tilde sign is dropped out, and $[R]$ denotes the combined concentration of the free radicals.

\section{SINGLE-STEP REACTION MECHANISM}

A well-known single-step reaction mechanism with equations for monomer conversion and heat diffusion ${ }^{21}$ was also considered for the comparison purpose. In this case the 2-D equations for the first-order reaction are

$$
\frac{d \alpha}{d t}=k_{1}(1-\alpha)
$$

where $\alpha$ is dimensionless conversion with zero value corresponding to pure monomer and 1 to pure polymer, The reaction rate $k_{1}$ has the usual Arrhenius temperature dependence

$$
k_{1}=A_{1} \exp \left(\frac{-E_{a}}{R_{g} T}\right)
$$

and the heat balance equation takes the form

$$
\frac{\partial T}{\partial t}=\kappa\left(\frac{\partial^{2} T}{\partial x^{2}}+\frac{\partial^{2} T}{\partial y^{2}}\right)-\frac{\Delta H}{\rho c_{p}} k_{1}(1-\alpha) .
$$

Here we assume that the only reaction taking place converts monomer into polymer. The preexponential factor and activation energy in this case are "effective" kinetic parameters of the process, representing all the reactions taking place in real systems. 
For purpose of testing the model the values of $E_{a}$ were varied in the range $14-30 \mathrm{kcal} \mathrm{mol}^{-1}$ and $A_{1}$ in the range $10^{5}-10^{12} \mathrm{~s}^{-1}$, respectively.

\section{NUMERICAL METHODS}

Finite difference numerical methods were used in our simulations. The system (16) $-(20)$ with initial conditions (12) and boundary conditions (13) and (14) was integrated on an adaptive space grid with a constant time step. In a typical wave front, variables such as the concentrations of the initiator, free radicals, and monomer, as well as the temperature change very slowly away from the reaction zone and extremely rapidly in the reaction zone. That is why an adaptive grid with the variable space step was used to maintain roughly the same number of grid points $(20-30)$ in the narrow moving reaction zone $(2-5 \mathrm{~mm}$ wide) to accurately resolve large gradients.

Ordinary differential equations describing kinetics of the process were solved using explicit Runge-Kutta methods of second or fourth order. Values of all variables whenever possible were taken from the upper time layer, i.e., the discrete solution on the current time step $n+1$ was used instead of values from previous time step $n$. Obviously, that could not be done for the temperature in the exponential temperature dependence of the reaction rates; its value was taken from the step $n$, i.e., the semi-implicit integrator was employed for the temperature. Iterations were performed for all variables using the updated temperature solution on each time step until the convergence of the solution was achieved. The convergence criterion was chosen to be the maximum scaled divergence for all variables on two consecutive iterations not to exceed $10^{-10}$

Semi-implicit finite difference schemes ${ }^{12}$ were used for linearization of Eqs. (20) and (23) on the discrete space grid. The system of linear equations with a tridiagonal matrix resulting from the finite difference approximation of the parabolic thermal conductivity Eqs. (20) and (23) was inverted using a modification of the Gauss method. The values of the temperature in the heat source term from the current time layer $n+1$ could not be used, however, without significant complications in the solution algorithm. Thus the values from the time step $n$ were used, and updated temperature values were substituted into all equations of the system for performing the iteration procedure described above.

The accuracy and convergence of the solution have been verified by consecutive divisions of the time step and comparing the results. The iterations continued until the convergence criterion was satisfied for all variables. Decreasing of the time step was used to eliminate large time scale dependence of the solution. At some critical value of the time step (usually in the range $10^{-4}-10^{-3} \mathrm{~s}$ ) the solutions cease to depend on it and are believed to represent the real system behavior.

The original adaptive grid algorithm ${ }^{12}$ was developed for the 1-D case. Based on it we developed the 2-D grid algorithm for use in our systems. It realizes dividing space steps according to custom defined criteria, i.e., inserts new points

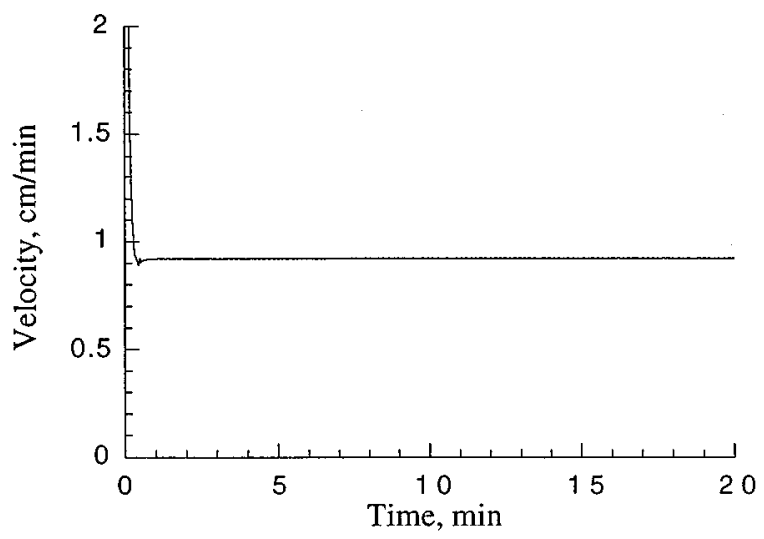

FIG. 1. Constant velocity front propagation: one-step reaction, $E_{a}$ $=18 \mathrm{kcal} \mathrm{mol}^{-1}, k_{1}=8 \times 10^{7} \mathrm{~s}^{-1}, T_{0}=300 \mathrm{~K}, \Delta T=200 \mathrm{~K}$,

in the regions of high gradients of given variables along with interpolation of variables' values and deletes points in the regions with small changes. The numerical scheme realized on the nonuniform space grid has the second order of approximation.

\section{RESULTS AND DISCUSSION}

\section{A. One-step reaction mechanism}

The effective activation energy $E_{a}$ of the polymerization reaction and the effective preexponent $A_{1}$ were used for the 1-D and 2-D simulations. The route to chaos as a sequence of period doubling bifurcations was observed as $E_{a}$ increased from $\neg 16-18 \mathrm{kcal} \mathrm{mol}^{-1} \quad$ (constant $\neg$ front $\neg$ velocity) to $28-30 \mathrm{kcal} \mathrm{mol}^{-1}$ (chaotic oscillations of the front velocity) in the 1-D case as had been previously shown by Matkowsky et $a .^{22,23}$ for solid-state combustion reactions. Figures 1-4 show the 1-D front velocity versus time for the same initial and adiabatic combustion temperatures as the Zeldovich number increases. The Zeldovich number ${ }^{16}$ is defined as

$$
Z=\frac{T_{m}-T_{0}}{T_{m}} \frac{E_{a, \mathrm{eff}}}{R_{g} T_{m}} . \neg
$$

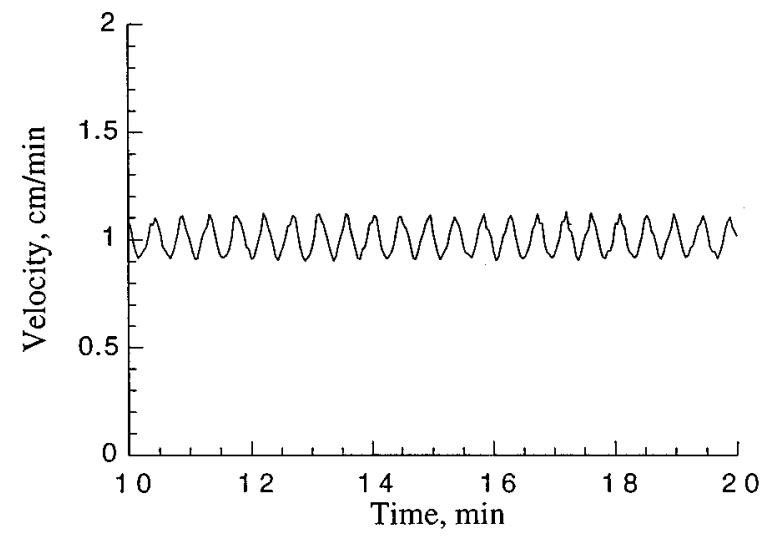

FIG. 2. Periodic oscillations, $E_{a}=20 \mathrm{kcal} \mathrm{mol}^{-1}, k_{1}=8 \times 10^{8} \mathrm{~s}^{-1}, Z=8$. 


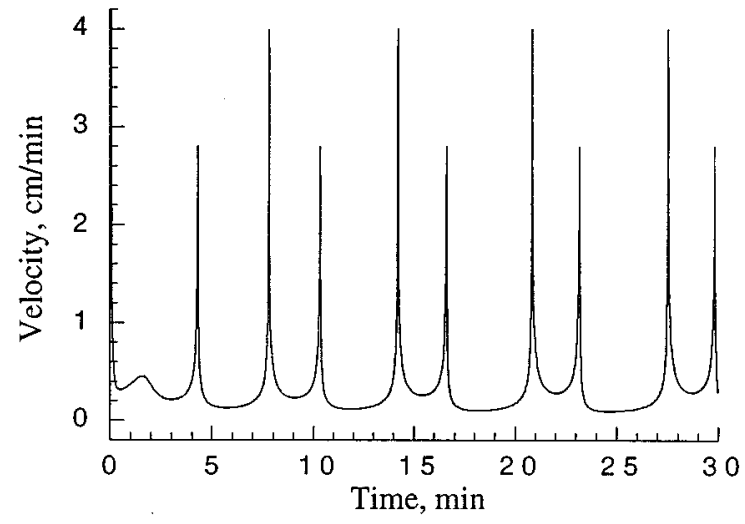

FIG. 3. Period two oscillations, $E_{a}=24 \mathrm{kcal} \mathrm{mol}^{-1}, k_{1}=8 \times 10^{9} \mathrm{~s}^{-1}, Z$ $=9.6$.

Here, $E_{a \text {,eff }}$ is the effective activation energy of the reaction, and $T_{m}$ is the maximum adiabatic temperature in the case of constant velocity front propagation. In case of oscillations the temperature in the front routinely exceeds the adiabatic temperature of combustion, balanced by the temperature dip behind the front. This is a specific feature of all periodic modes obtained numerically and supported by the experimental measurements in our lab (Fig. 5).

Strange as it might seem the phenomenon of superadiabatic combustion temperature has never been explained in the literature, although it can serve as an experimental indicator of any thermal instability beyond the Hopf bifurcation as it never appears in stable combustion. The difference in characteristic times of the chemical reaction and thermal diffusion at initial and combustion temperatures (as a result of exponential temperature dependence of the reaction rates) at some point leads to preheating of the initial reactive mixture ahead of the temperature wave without significant reaction in this zone. The following thermal "explosion" on the wave front results in apparent exceeding of the adiabatic combustion temperature because it occurs at effectively higher initial temperature. The energy conservation law, however, is not violated here since this excessive heat release is localized in the wave front and balanced by lower than adiabatic tem-

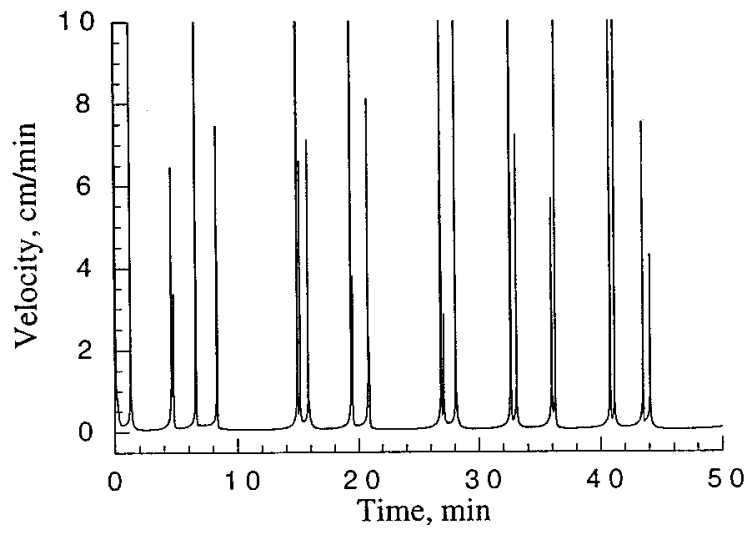

FIG. 4. Chaotic oscillations, $E_{a}=30 \mathrm{kcal} \mathrm{mol}^{-1}, k_{1}=8 \times 10^{13} \mathrm{~s}^{-1}, Z=12$.

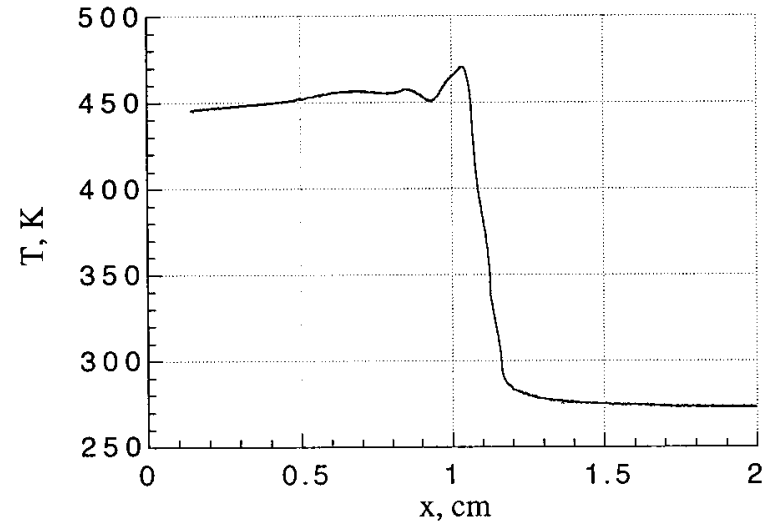

FIG. 5. Experimental temperature profile for methacrylic acid with 2 wt. \% benzoyl peroxide system exhibiting a single head spin mode, $T_{0}=273 \mathrm{~K}$, $D=22 \mathrm{~mm}$.

perature behind the "explosion." When this structure eventually goes through a relaxation stage (as the temperature gradient is extremely high, and some time is needed for heat to diffuse and trigger the reaction in the cold mixture), the temperature on and behind the wave front equilibrates, and the superadiabatic peak disappears. The critical difference in characteristic times between the chemical reaction and diffusion for the phenomenon to appear is determined by the thermal diffusivity coefficient and the Zeldovich number at which the Hopf bifurcation occurs.

The superadiabatic temperature $T_{s}$ does not stay the same during the oscillation period being the highest as the front velocity $\nu$ reaches its maximum and the lowest (equal to adiabatic temperature) as $\nu$ reaches its minimum [compare, e.g., Figs. 9(c) and 10(c) for the three-step model; for the one-step model the temperature profiles remain basically the same]. That is why the maximum front temperature cannot be used as $T_{m}$ for determining $Z$, and the established equilibrium temperature behind the front is used for this purpose. In Figs. 9(c) and 10(c) this equilibrium temperature lies in the range $x=4-7 \mathrm{~cm}$, higher temperatures around $x=0$ are the result of prolonged ignition and adiabatic boundary conditions.

Planar 2-D front velocity oscillations or one and two head periodic modes were observed in our simulations depending on the tube diameter, effective activation energy (Zeldovich number) and initial conditions. Shown in Figs. 6(a) and 6(b) are two possible patterns for one and two head mode propagation that can coexist at the same conditions. The two head mode with heads moving in the same direction was never observed in 2-D simulations, although it can exist if periodic boundary conditions (i.e., in the case of a front propagating on the surface of the cylinder) are used. ${ }^{12}$ Figures 7(a) and 7(b) show numericallyalculated sample 2-D temperature fields corresponding to the patterns in Fig. 6. In Fig. 7(a) the head moves from left to right leaving hightemperature trace behind, and in Fig. 7(b) two heads are moving in opposite directions near the collision at the tube axis. 


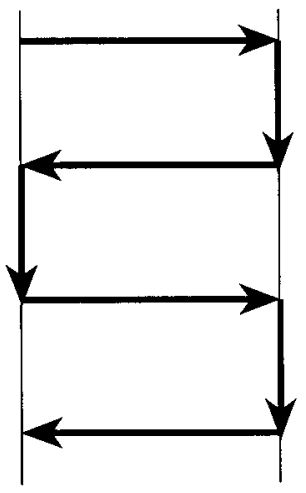

(a)

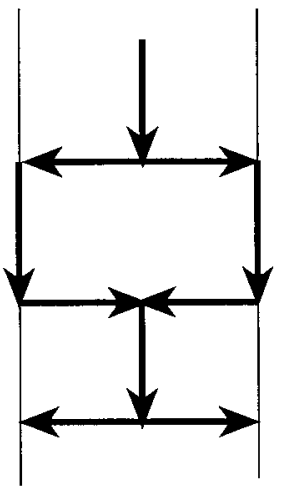

(b)
FIG. 6. Proposed patterns of propagation of one (a) and two (b) head modes in the 2-D case. Arrows show the direction of the head(s) movement along the wave front moving downward.

\section{B. 2-D stability diagram}

Our approximate analytical stability analysis of the 2-D problem (following the 2-D analysis by Makhviladze and Novozhilov ${ }^{24}$ resulted in $k-Z$ stability diagram where $k$ is a wave number) shows the neutral stability boundaries between different modes arising in 2-D fronts on the $D-Z$ diagram (Fig. 8). Here $D$ is the tube diameter. Crossing any stability boundary while moving up or right in the parameter
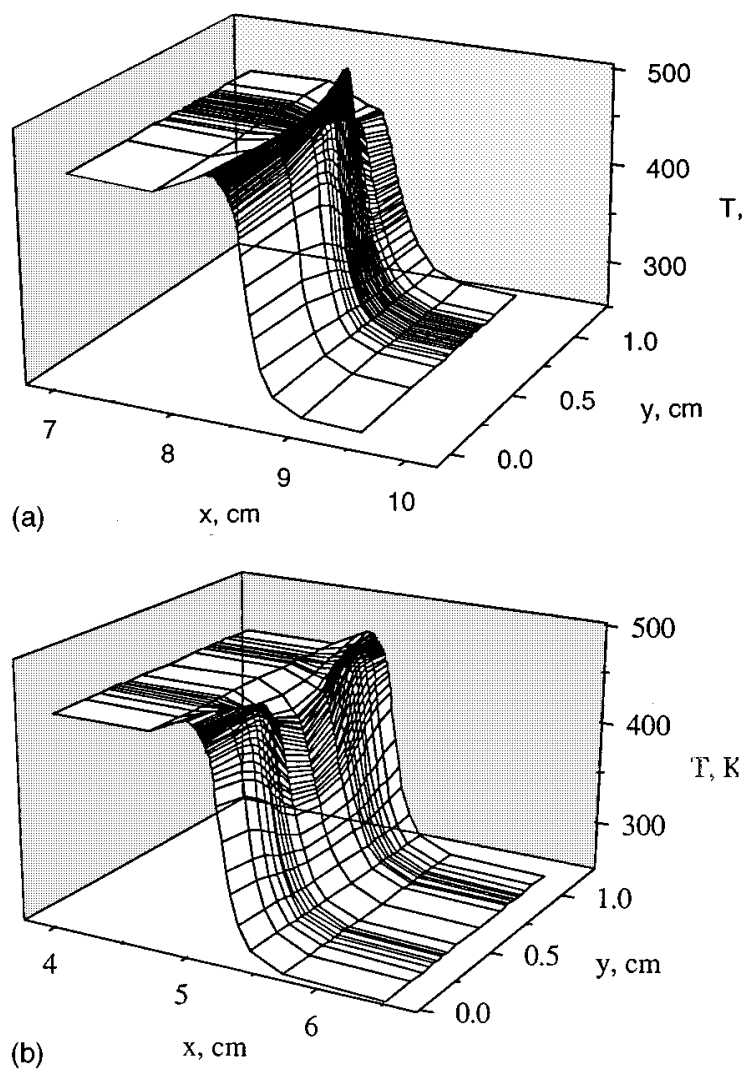

FIG. 7. Sample 2-D temperature profiles with one head (a) and two heads (b) for one-step reaction mechanism. The tube diameter $D=12 \mathrm{~mm}, T_{0}$ $=253 \mathrm{~K}, Z=9.7$.

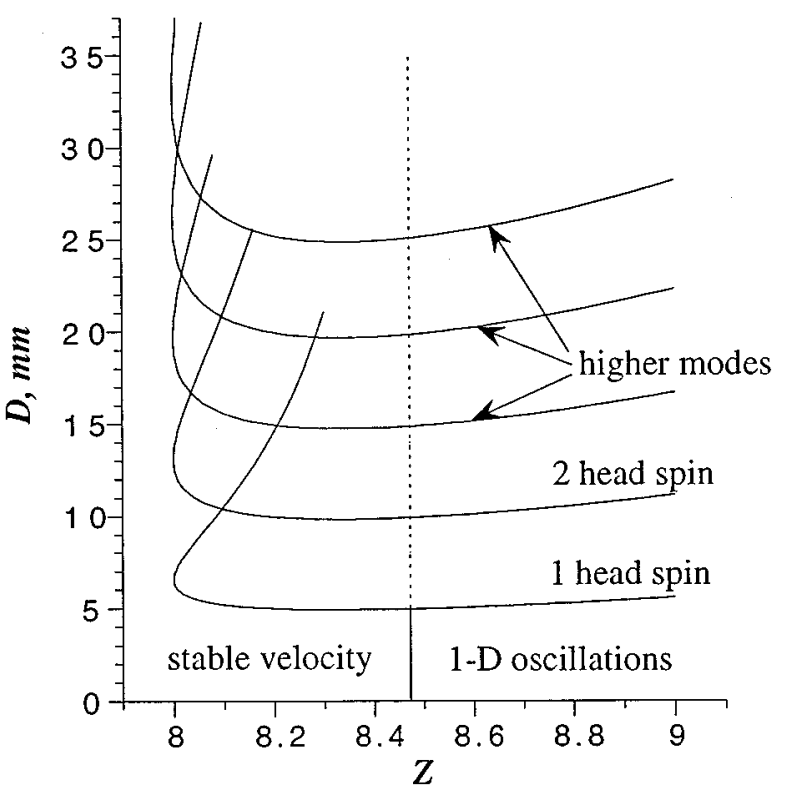

FIG. 8. $D-Z$ stability diagram for one-step reaction, $E_{a}=18 \mathrm{kcal} \mathrm{mol}^{-1}$, $k_{1}=11.58 \times 10^{7} \mathrm{~s}^{-1}, \kappa=0.001 \mathrm{~cm}^{2} \mathrm{~s}^{-1}, \Delta T=200 \mathrm{~K}$.

space results in the appearance of the denoted mode. As seen from the figure, for sufficiently small Zeldovich numbers only constant velocity wave fronts can be observed. As $Z$ increases planar front velocity oscillations are the case if the tube diameter is small. These planar oscillations can exhibit period doubling as $Z$ increases further. We demonstrated it earlier for the 1-D model.

For larger tube diameters one head, two head, and higher nonplanar modes (multiple heads, standing waves, etc.) appear as $D$ increases. In the regions where stability zones for different modes overlap, bistability was observed depending on the initial conditions. For example, for $Z>8.48$ and 5 $<D<10 \mathrm{~mm}$ planar oscillations and one head mode are expected to coexist. Indeed, the one head mode is observed only if the perturbation of the planar initial conditions is large enough, otherwise planar oscillations are sustained until the front reaches the bottom of the tube.

\section{Three-step reaction mechanism}

Numerical simulations showed that results for the threestep and one-step reaction kinetics significantly differ with respect to the front stability and the final product distribution. From the steady-state approximation of the free radical concentration in the reaction zone ${ }^{25}$ the effective activation energy can be calculated as

$$
E_{a, \mathrm{eff}}=E_{a, p}+\frac{E_{a, i}-E_{a, d}}{2}
$$

and the effective preexponent as

$$
\left.A_{\mathrm{eff}}=A_{p} \sqrt{\frac{2 f A_{d}}{A_{t}}} \cdot\right\urcorner
$$



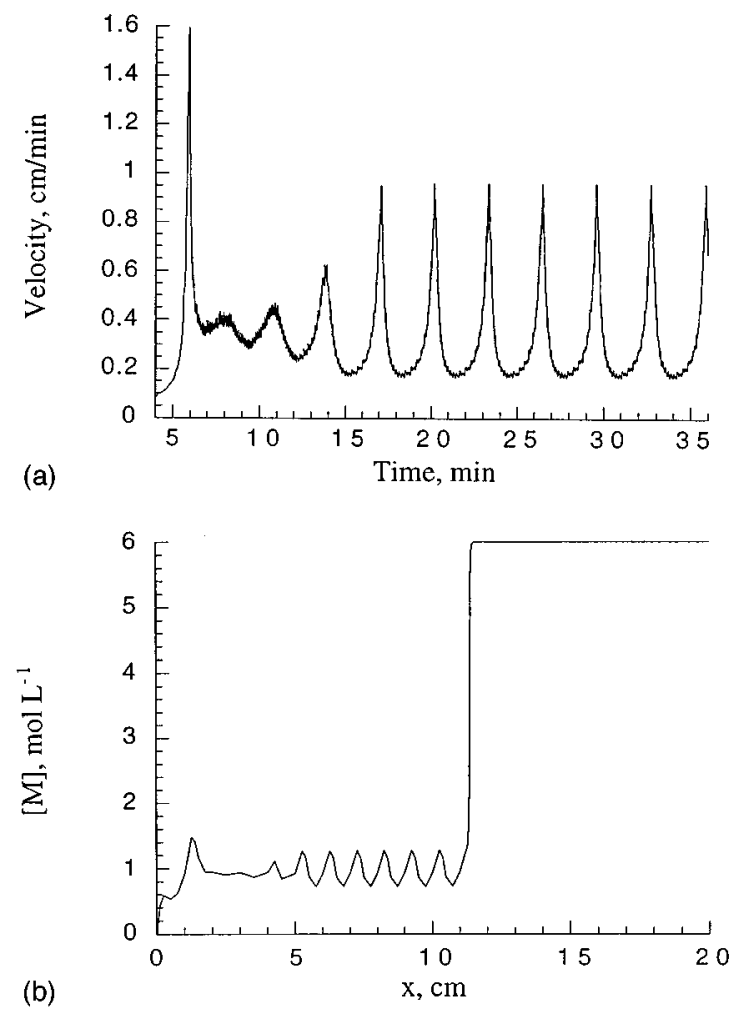
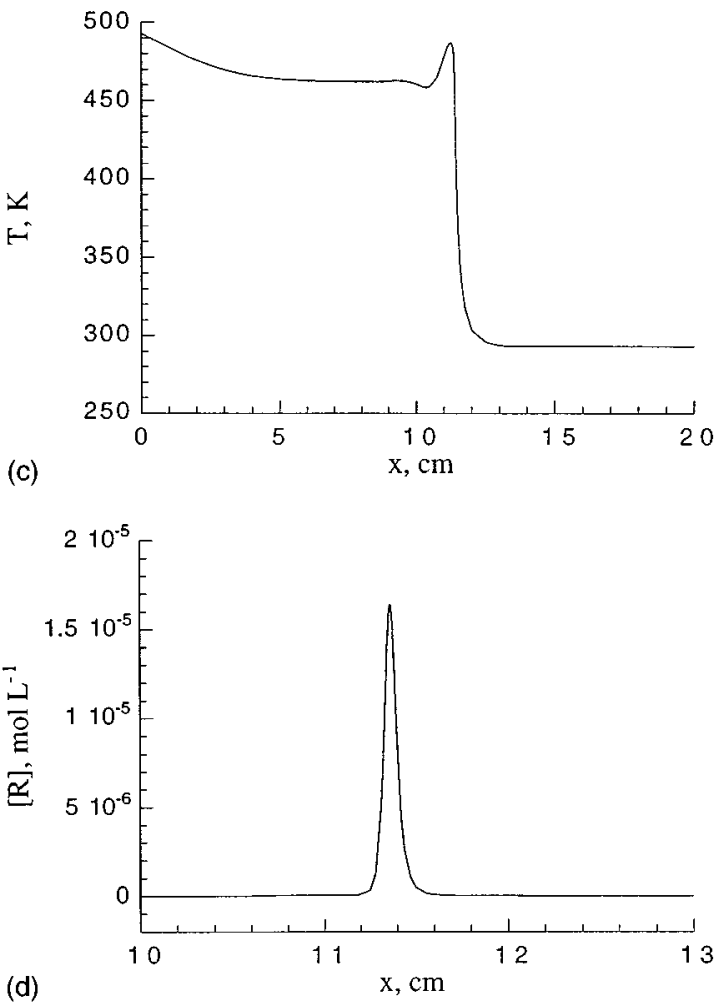

FIG. 9. (a) Front velocity versus time, $E_{a, \text { eff }}=24.15 \mathrm{kcal} \mathrm{mol}^{-1}, k_{\text {eff }}=3.7 \times 10^{11} \mathrm{~s}^{-1},[I]_{0}=0.02 \mathrm{M}, Z=9.64$ (periodic oscillations). (b) The monomer concentration profile at $t=36 \mathrm{~min}$. (c) The temperature profile at $t=36 \mathrm{~min}$ (the highest point of the front velocity oscillation). (d) The free radical concentration profile at $t=36 \mathrm{~min}$.

In this case the 1-D solutions for the three-step mechanism exhibit period doubling bifurcations at higher effective activation energies and correspondingly higher Zeldovich numbers [Figs. 9(a) and 10(a)] compared to the one-step mechanism (Figs. 2 and 3). The kinetic constants for Fig. 9 are

$$
\begin{aligned}
& \left.E_{a, d}=30.0 \mathrm{kcal} \mathrm{mol}^{-1},\right\urcorner A_{d}=4.0 \times 10^{12} \mathrm{~s}^{-1}, \\
& \left.E_{a, p}=10.5 \mathrm{kcal} \mathrm{mol}^{-1},\right\urcorner A_{p}=1.0 \times 10^{9} \mathrm{~s}^{-1} \mathrm{M}^{-1}, \\
& \left.E_{a, t}=0.7 \mathrm{kcal} \mathrm{mol}^{-1},\right\urcorner \quad A_{t}=3.0 \times 10^{7} \mathrm{~s}^{-1} \mathrm{M}^{-1},
\end{aligned}
$$

and for Fig. 10:

$$
\begin{aligned}
& \left.E_{a, d}=30.0 \mathrm{kcal} \mathrm{mol}^{-1},\right\urcorner A_{d}=4.0 \times 10^{12} \mathrm{~s}^{-1}, \\
& \left.E_{a, p}=11.5 \mathrm{kcal} \mathrm{mol}^{-1},\right\urcorner A_{p}=5.0 \times 10^{9} \mathrm{~s}^{-1} \mathrm{M}^{-1}, \\
& \left.E_{a, t}=0.7 \mathrm{kcal} \mathrm{mol}^{-1},\right\urcorner \quad A_{t}=3.0 \times 10^{7} \mathrm{~s}^{-1} \mathrm{M}^{-1} .
\end{aligned}
$$

More complicated kinetics in this case gives rise to the effects that could not be accounted for in the one-step model. First of all the complete conversion of the monomer is never observed in the three-step model and experimentally ${ }^{18}$ compared to the one-step case where the conversion is always $100 \%$. Figure 9(b) shows the trace of the unreacted monomer behind the front, and its concentration oscillates in phase with the front velocity oscillations (the solution behind the front does not seem well resolved only because the adaptive grid algorithm does not keep too many points behind the front where there are no high gradients of important vari- ables). When the front velocity reaches a maximum the monomer conversion is the lowest and vice versa. The reason for this is that during the temperature surge the initiator is being quickly burnt out in the front, and the free radicals are no longer produced. That leads a lower monomer conversion compared to the periods of slowdowns when the lower temperature allows for longer times of the initiator consumption and longer free radical lifetimes. It also should lead to a lower degree of polymerization during these temperature surges. Figures 9(c) and 9(d) show the temperature and free radical profiles at the same time as the monomer concentration profile in Fig. 9(b). The same holds for the period two front velocity oscillations shown in Fig. 10(a) and corresponding monomer, temperature, and free radical concentration profiles in Figs. 10(b)-10(d) where the period two oscillations of the monomer conversion along the tube are clearly defined. In Figs. 9(c) and 10(c) the temperature is constant behind the front except in the area close to $x=0$ where it is higher because of the adiabatic boundary conditions and the ignition temperature $T_{i}$ which was set to be higher than combustion temperature for specified conditions.

As seen from the figures the same modes are observed at higher Zeldovich numbers for the three-step mechanism than for the one-step mechanism. It should be noted that for the three-step mechanism corresponding Zeldovich numbers were calculated using lower than adiabatic combustion temperatures reflecting less than $100 \%$ conversion. This is spe- 

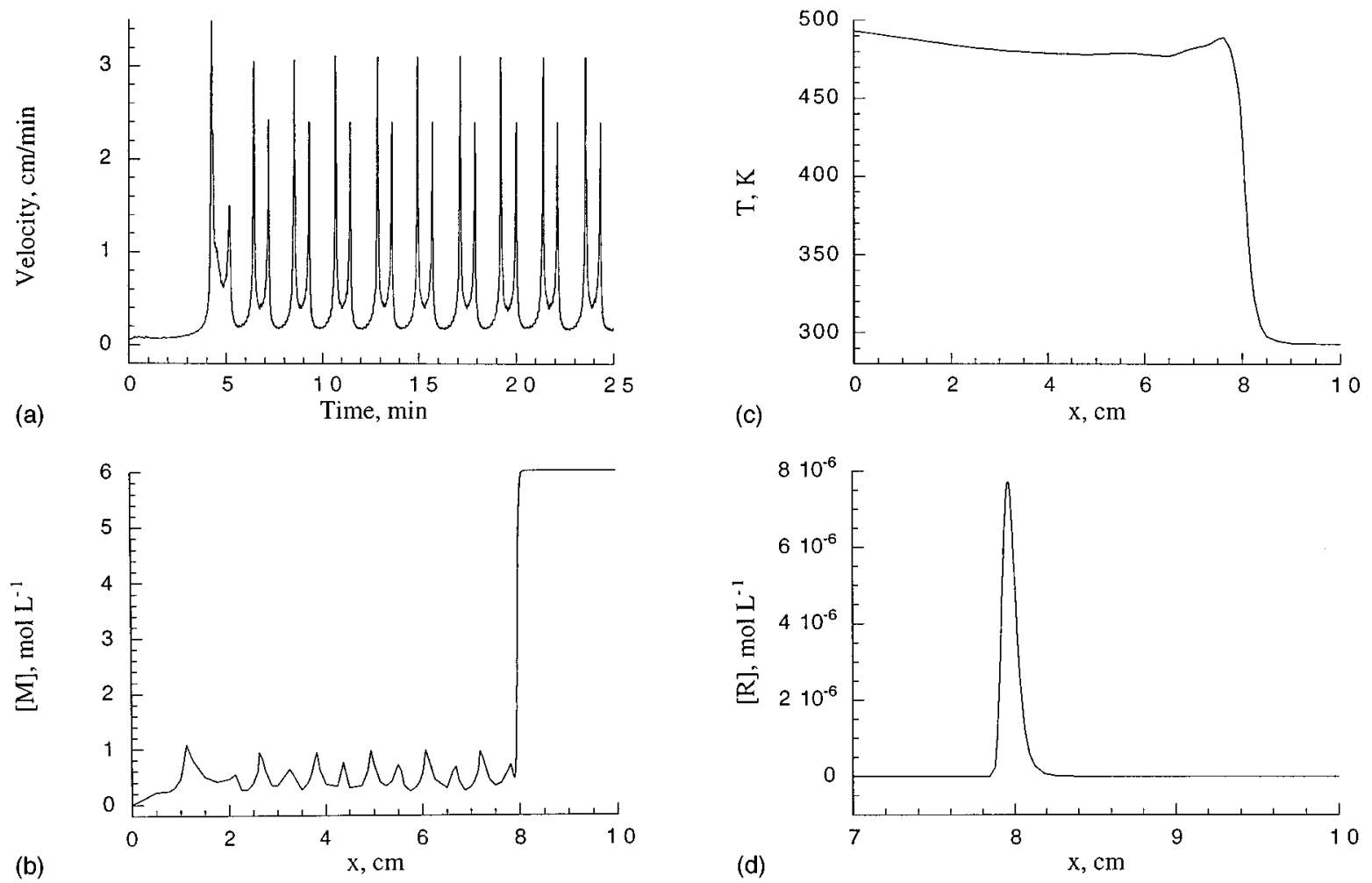

FIG. 10. (a) Front velocity versus time. $E_{a \text {,eff }}=25.15 \mathrm{kcal} \mathrm{mol}^{-1}, k_{\text {eff }}=1.8 \times 10^{12} \mathrm{~s}^{-1},[I]_{0}=0.02 \mathrm{M}, Z=10.25$ (period two oscillations). (b) The monomer concentration profile at $t=16 \mathrm{~min}$. (c) The temperature profile at $t=16 \mathrm{~min}$ (close to the lowest point of the front velocity oscillation). (d) The free radical concentration profile at $t=16 \mathrm{~min}$.

cific for the experimental data and the three-step mechanism involving the initiator and free radicals. Thus, the three-step reaction mechanism always tends to produce more stable solutions in the Zeldovich number sense, i.e., for the same $Z$ (assuming the steady-state approximation is valid) the threestep solutions are expected to exhibit "lower" modes compared to the one-step solutions. Here the "lower" modes mean, e.g., period one oscillations compared to period two and constant velocity wave front compared to periodic oscillations.

\section{2-D simulations}

The same trend as for the 1-D case is observed in the 2-D simulations. Many 2-D nonplanar periodic modes can be obtained in the numerical experiments only for larger tube diameters (see Fig. 8). Higher Zeldovich numbers are needed to observe nonplanar periodic modes compared to the singlestep reaction mechanism. Increasingly complex behavior is observed as $Z$ increases. The 2-D temperature profiles in Figs. 11(a)-11(e) calculated for the parameters producing period two 1-D oscillations [see Fig. 10(a)] with an asymmetric initial temperature perturbation demonstrate the point. The complicated asymmetric pattern with two heads similar to the pattern in Fig. 6(b) was observed. The difference is that two heads moving in opposite directions appear not in the center of the tube but somewhat closer to the right wall. When these heads move around and collide, the collision occurs near the left wall because the velocities of the heads are approximately equal. After the collision the heads disappear until the next hot spot is formed again near the right wall and the cycle repeats. This pattern appeared to be stable, however, for axisymmetric initial conditions a symmetric pattern like in Fig. 6(b) was observed.

We note that when the head reflects from the wall, its maximum temperature $T_{s}$ does not significantly exceed $T_{m}$ but as long as it moves along the front its $T_{s}$ increases up to 30-50 K higher than $T_{m}$. We concluded from the results of our 1-D simulations that during the oscillation period high front velocities with very high $T_{s}$ in the front result in lower conversions. The same behavior was expected for the 2-D model. Due to asymmetry of the propagation pattern obtained for asymmetric initial conditions (Fig. 11) the maximum head temperature was the highest during its movement from right to left along the front moving downward. It should have left low monomer conversion traces in the formed product. Indeed, the traces of low conversion following the head paths from right to left were observed, and they are shown in Fig. 12(a) which represents the 2-D monomer profile in and behind the reaction zone [compare to Fig. 10(b)]. The contour plot in Fig. 12(b) corresponding to Fig. 12(a) clearly shows the right to left direction of these traces as the front moved downward.

\section{CONCLUSIONS}

Periodic modes play a significant role in propagating polymerization fronts dynamics and also affect properties of 

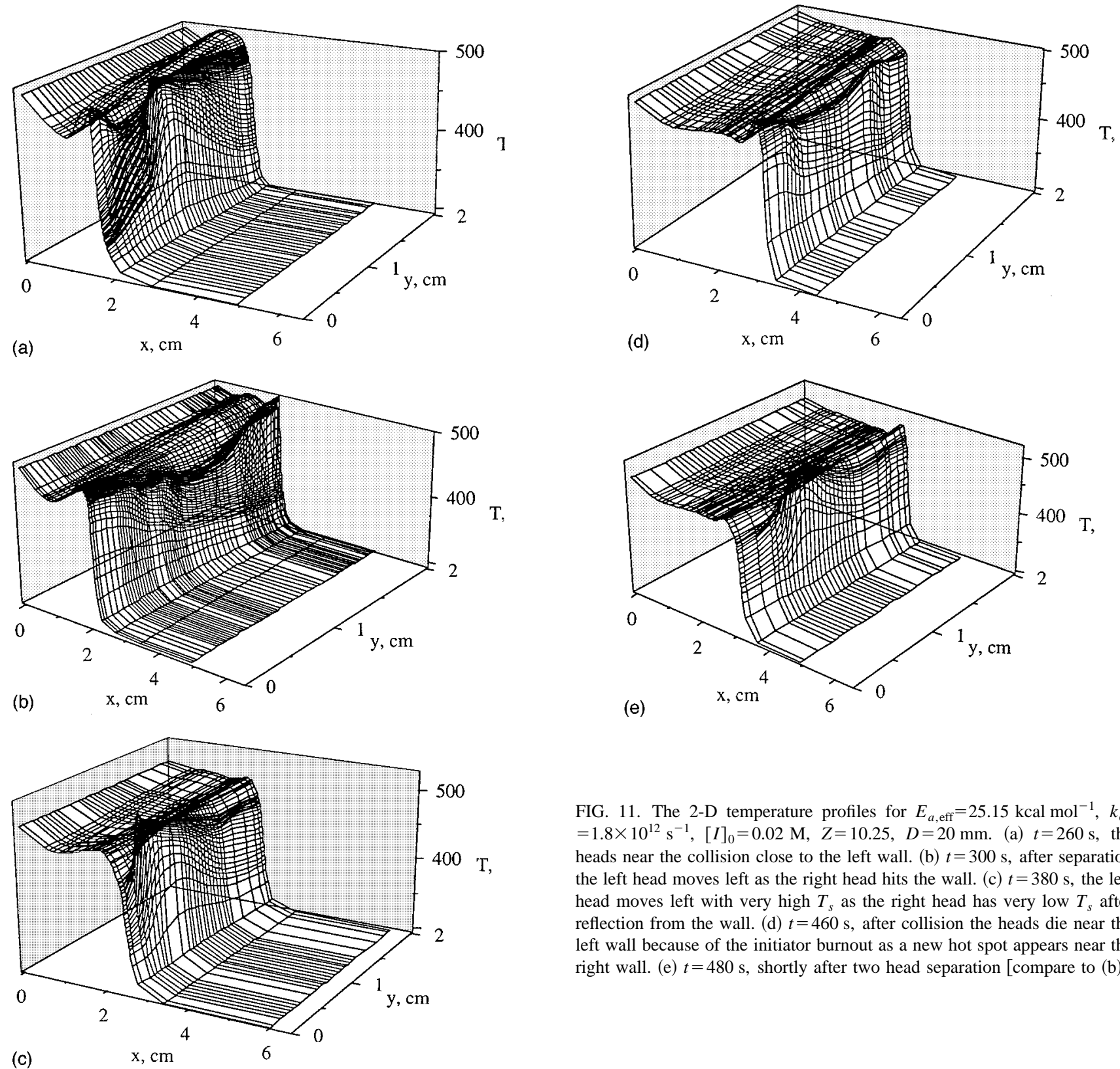

(e)

FIG. 11. The 2-D temperature profiles for $E_{a \text {,eff }}=25.15 \mathrm{kcal} \mathrm{mol}^{-1}, k_{\text {eff }}$ $=1.8 \times 10^{12} \mathrm{~s}^{-1},[I]_{0}=0.02 \mathrm{M}, Z=10.25, D=20 \mathrm{~mm}$. (a) $t=260 \mathrm{~s}$, the heads near the collision close to the left wall. (b) $t=300 \mathrm{~s}$, after separation the left head moves left as the right head hits the wall. (c) $t=380 \mathrm{~s}$, the left head moves left with very high $T_{s}$ as the right head has very low $T_{s}$ after reflection from the wall. (d) $t=460 \mathrm{~s}$, after collision the heads die near the left wall because of the initiator burnout as a new hot spot appears near the right wall. (e) $t=480 \mathrm{~s}$, shortly after two head separation [compare to (b)].

the final product. In this paper we demonstrated that the effective activation energy of the polymerization reaction, the Zeldovich number, and the tube diameter are some of the bifurcation parameters determining the stability of propagating polymerization fronts, and also offered an explanation of the phenomenon of superadiabatic combustion temperature. Other factors such as the initiator concentration, reaction enthalpy, etc affect the velocity of the front rather than its stability. As was found, stability of the frontal mode for the one-step reaction mechanism does not differ for 1-D and 2-D cases. For the three-step reaction mechanism 2-D solutions are more stable than corresponding 1-D ones in respect to appearance of nonplanar periodic modes contrary to the accepted result ${ }^{24}$ that 2-D solutions are always less stable. In our case it can be explained by the stabilizing effect of the

tube walls. Higher Zeldovich numbers (i.e., higher effective activation energies or lower initial temperatures) are needed for the existence of nonplanar periodic or planar oscillatory modes in the 2-D reactor with walls than for the existence of oscillations in the 1-D case.

Comparing the one-step and three-step kinetics we conclude that the more realistic three-step reaction mechanism shifts up and right the stability boundaries on the $D-Z$ diagram compared to the simplified one-step mechanism, because for the three-step mechanism the nonplanar periodic modes are not observed for the tube diameters and Zeldovich numbers resulting in such modes for the one-step mechanism. The boundary between stable front velocity and 1-D oscillations stays, and it is not surprising because this boundary does not depend on the tube diameter. Nonplanar modes 

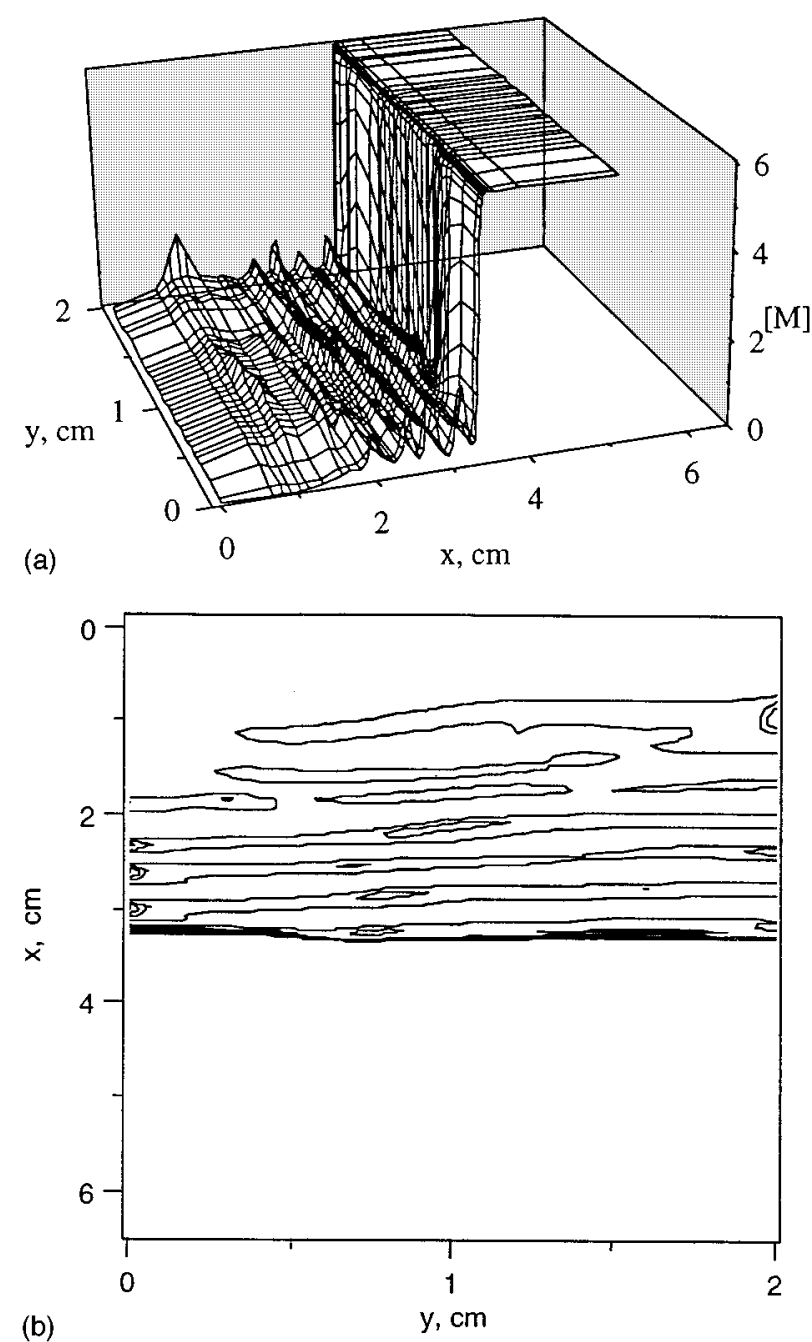

FIG. 12. The 2-D monomer concentration profile (a) and its contour plot (b) at $t=480 \mathrm{~s}$ for the same parameter values as in Fig. 11 .

also are not observed if the tube diameter is sufficiently small.

\section{ACKNOWLEDGMENTS}

We thank Dr. Vit. Volpert and Dr. Vl. Volpert for valuable ideas and discussion. We also gratefully acknowledge
N. Surkov (Institute of Chemical Physics, Chernogolovka, Russia) for generous permission to use his original 1-D adaptive grid algorithm. This work was supported by the National Science Foundation (Grant No. CTS-9319175 and the Mississippi EPSCoR program).

${ }^{1}$ A. G. Merzhanov, A. K. Filonenko, and I. P. Borvinskaya, Sov. Phys. Dokl. 208, 122 (1973).

${ }^{2}$ A. G. Merzhanov, Archiv. Comb. 1, 23 (1981).

${ }^{3}$ N. M. Chechilo, R. J. Khvilivitskii, and N. S. Enikolopyan, Dokl. Akad. Nauk SSSR 204, 1180 (1972).

${ }^{4}$ N. M. Chechilo and N. S. Enikolopyan, Dokl. Phys. Chem. 214, 174 (1974).

${ }^{5}$ J. A. Pojman, J. Am. Chem. Soc. 113, 6284 (1991).

${ }^{6}$ I. P. Nagy, L. Sike, and J. A. Pojman, J. Am. Chem. Soc. 117, 3611 (1995).

${ }^{7}$ A. M. Khan and J. A. Pojman, Trends Polym. Sci. 4, 253 (1996).

${ }^{8}$ J. A. Pojman, R. Craven, A. Khan, and W. West, J. Phys. Chem. 96, 7466 (1992).

${ }^{9}$ J. A. Pojman, V. M. Ilyashenko, and A. M. Khan, Physica D 84, 260 (1995).

${ }^{10}$ J. A. Pojman, V. M. Ilyashenko, and A. M. Khan, J. Chem. Soc. Faraday Trans. 92, 2825 (1996).

${ }^{11}$ A. S. Babadzhanyan, V. A. Volpert, V. A. Volpert, S. P. Davtyan, and I. N. Megrabova, Combust. Explos. Shock Waves 24, 711 (1988).

${ }^{12}$ V. A. Volpert, V. A. Volpert, S. P. Davtyan, I. N. Mergabova, and N. F. Surkov, SIAM (Soc. Ind. Appl. Math.) J. Appl. Math. 52, 368 (1992)

${ }^{13}$ S. E. Solovyov, V. A. Volpert, and S. P. Davtyan, SIAM (Soc. Ind. Appl. Math.) J. Appl. Math. 53, 907 (1993).

${ }^{14}$ J. B. Zeldowitsch and D. A. Frank-Kamenetzki, Acta Physicochim. URSS IX, 341 (1938).

${ }^{15}$ D. A. Frank-Kamenetsky, Diffusion and Heat Exchange in Chemical Kinetics (Princeton University Press, Princeton, NJ, 1955).

${ }^{16}$ Y. B. Zeldovich, G. I. Barenblatt, V. B. Librovich, and G. M. Makhviladze, The Mathematical Theory of Combustion and Explosions (Consultants Bureau, New York, 1985).

${ }^{17}$ B. V. Novozhilov, Dokl. Akad. Nauk SSSR 141, 151 (1961).

${ }^{18}$ J. A. Pojman, J. Willis, D. Fortenberry, V. Ilyashenko, and A. Khan, J. Polym. Sci. Part A: Polym Chem. 33, 643 (1995).

${ }^{19}$ J. A. Pojman, J. R. Willis, A. M. Khan, and W. W. West, J. Polym. Sci. Part A: Polym Chem. 34, 991 (1996).

${ }^{20}$ A. A. Gridnev and S. D. Ittel, Macromolecules 29, 5864 (1996).

${ }^{21}$ B. B. Khanukaev, M. A. Kozhushner, and N. S. Enikolopyan, Combust. Explos. Shock Waves 10, 562 (1974).

${ }^{22}$ B. J. Matkowsky and G. I. Sivashinsky, SIAM (Soc. Ind. Appl. Math.) J. Appl. Math. 35, 465 (1978).

${ }^{23}$ A. Bayliss and B. J. Matkowsky, J. Comput. Phys. 71, 147 (1987).

${ }^{24}$ G. M. Makhviladze and B. V. Novozhilov, Prikl. Mech. Techn. Fiz. No. 5 51 (1971).

${ }^{25}$ G. Odian, Principles of Polymerization (Wiley, New York, 1981), Vol. 1, p. 261. 
Chaos is copyrighted by AIP Publishing LLC (AIP). Reuse of AIP content is subject to the terms at: http://scitation.aip.org/termsconditions. For more information, see http://publishing.aip.org/authors/rights-and-permissions. 